\title{
Distribution et caractérisation par fluorescence de la matière organique dissoute dans les eaux de la Manche centrale
}

\author{
M. Monaïm ISMAILI ${ }^{a}$, Colette BELIN ${ }^{\text {b }}$, Michel LAMOTTE ${ }^{b}$, Hervé TEXIER ${ }^{\text {a }}$ \\ ${ }^{a}$ Laboratoire de géologie marine, université de Caen, 14032 Caen cedex, France \\ ${ }^{\text {b }}$ Laboratoire de photophysique et photochimie moléculaires, université Bordeaux-I, 33405 Talence, France
}

(Reçu le 24/06/97, révisé le 02/07/98, accepté le 02/07/98)

\begin{abstract}
Distribution and characterisation by fluorescence of the dissolved organic matler within the central Channel waters. Intensity and spectral characteristics of the fluorescence of water samples collected along the transect Cherbourg-Isle of Wight during four cruises for excitations at 370, 313 and $270 \mathrm{~nm}$ have been investigated within the framework of the FluxManche II program. Seasonal and spatial differences appeared to reflect on the one hand, variations in terrestrial inputs, and on the other hand, waters masses structuring. The observed linear variation of the fluorescence intensity with salinity indicates a gradual dilution of the continental humic material in going from the coasts to the central part of the English Channel. The largest signal was observed for the English coastal waters in agreement with important terrestrial inputs from the Solent river. While the fluorescence intensity was not found to be correlated with the DOC, it shows a good correlation with the nutrients. This result could indicate both a supply from terrestrial inputs and an almost simultaneous autochtonous regeneration. As similarly reported for waters in the eastern part of the French coaslal zone, excitation at $313 \mathrm{~nm}$ gives evidence for the presence of two classes of fluorescent dissolved organic matter. These two fluorescent components are indicative of the simultaneous presence of continental humic substances and substances whose assignment to marine humic substances or to heterotrophic related substances are still an open question. As compared to the eastern part of the Channel along the French coasts, excitation at $270 \mathrm{~nm}$ indicates a lower content of protein-like compounds which may be related to a lower biological activity along the transect or may be due to the fact that the samplings were made out of the phytoplanktonic bloom period (April-May 1995). (C) Elsevier, Paris
\end{abstract}

\section{spectrofluorimetry / dissolved organic matter / English Channel / tracer of water mass / nutrient}

Résumé - Quatre campagnes de prélèvements des eaux du transect Cherbourg - île de Wight en Manche centrale ont été menées dans le cadre du programme FluxManche II. La matière organique dissoute fluorescente a été étudiée par spectrofluorimétrie sous le double aspect quantitatif et qualitatif. La fluorescence naturelle des eaux étudiées montre des variations spatiales et saisonnières en relation avec l'influence des apports terrestres et avec la structuration des masses d'eaux. Des relations linéaires négatives entre la fluorescence et la salinité suggèrent une dilution graduelle du matériel humique continental des côtes vers le centre de la Manche. Le signal le plus élevé est observé dans les eaux côtières anglaises en relation avec l'influence des apports du Solent. Alors que la fluorescence diffère entre le matériel humique et la matière organique dissoute globale, en raison principalement de cinétiques d'évolution différentes, elle est liée aux sels nutritifs $\left(\mathrm{Si}, \mathrm{NO}_{3}^{-}, \mathrm{PO}_{4}^{3-}\right)$ par des relations linéaires positives indiquant des apports terrestres concomitants et une régénération commune autochtone de ces deux paramètres. L'excitation à $313 \mathrm{~nm}$ met en évidence deux composantes spectrales; l'une est attribuée aux substances humiques continentales, l'autre semble caractéristique du milieu marin. L'origine et les mécanismes aboutissant à la formation de cette composante fluorescente marine restent à établir. Par comparaison à la Manche orientale, le long des côtes françaises, l'excitation à $270 \mathrm{~nm}$ indique une faible teneur en composés de type protéinique, probablement liée à la faible activité biologique le long du transect ou au fait que les prélèvements ont eu lieu en dehors (avril-mai 1995) de la période de floraison phytoplanctonique. (C) Elsevier, Paris

spectrofluorimétrie / matière organique dissoute / Manche / traceur de masse d'eau / nutriment 


\section{INTRODUCTION}

Il est admis que la fluorescence des eaux naturelles donne lieu au moins à deux bandes principales de fluorescence, l'une dans le domaine 300-350 nm environ, attribuée à du matériel protéinique ou à des composés phénoliques, l'autre vers 390-470 nm attribuée aux substances humiques [7]; de Souza-Sierra et al. [10] suggèrent que la fluorescence des eaux naturelles excitée entre 310$320 \mathrm{~nm}$ peut être modélisée par la présence de deux familles de fluorophores dont l'une, émettant vers les courtes longueurs d'ondes $(\lambda \mathrm{em} \approx 390 \mathrm{~nm}$ ), est considérée comme la plus abondante dans les eaux marines et caractérise la signature spectrale de ces masses d'eaux. Coble [7] a mis en évidence dans des échantillons d'eau côtière, une composante : $\lambda \mathrm{ex}=310-320 ; \lambda \mathrm{em}=380$ 400 qui pourrait être d'origine biologique purement marine. Cette hypothèse déjà proposée par de SouzaSierra et al. [10] est à rapprocher de la suggestion de Determann et al. [11], selon laquelle une source importante des substances dissoutes fluorescentes dans le milieu marin serait liée à la dégradation d'exsudats phytoplanctoniques.

De l'ensemble de ces observations, il ressort qu'une excitation dans le domaine $300-320 \mathrm{~nm}$ est la plus apte à différencier la matière organique dissoute dans les eaux naturelles en fonction de son origine. Cette différenciation spectrale n'apparaît pas aussi clairement avec d'autres longueurs d'ondes d'excitation, et notamment avec $\lambda$ ex dans le domaine $360-380 \mathrm{~nm}$, qui s'avère mieux adapté pour estimer quantitativement la teneur globale en substances humiques (SH) qui représente généralement la partie la moins labile de la matière organique dissoute (MOD) [12].

Dans le présent travail, une étude fluorimétrique des eaux a été effectuée en Manche centrale entre Cherbourg et l'île de Wight. L'objectif était d'estimer les possibilités qu'offre la spectroscopie de fluorescence pour caractériser et différencier les masses d'eaux, en relation avec l'origine et la nature de la matière organique fluorescente dissoute qu'elles contiennent. Les variations latérales et verticales des intensités de fluorescence pour $\lambda$ ex $=370 \mathrm{~nm}$, ainsi que les profils des spectres pour $\lambda \mathrm{ex}=313 \mathrm{~nm}$ et, pour certains échantillons, la présence d'une bande $(\lambda \mathrm{em}=300-320 \mathrm{~nm})$ associée à du matériel protéinique pour $\lambda \mathrm{ex}=270 \mathrm{~nm}$ sont discutés en relation avec d'autres paramètres tels que la salinité, la chlorophylle $a$, le carbone organique dissous (COD) et les sels nutritifs.

\section{MATÉRIELS ET MÉTHODES}

\subsection{Site d'étude}

La Manche, par sa configuration physique, est un bassin épicontinental, ce qui signifie que la plupart des mécanismes de dynamique interne des océans en sont absents [19]. Elle constitue une zone de transit d'eaux atlantiques vers la Mer du Nord. En raison de la dynamique macrotidale et du forçage météorologique, elle est le siège d'importantes remises en suspension et d'un brassage quasi permanent de la colonne d'eau. La circulation des masses d'eaux sur le transect Cherbourg-Wight est composée d'une veine d'eau centrale et de tourbillons latéraux, notamment dans les eaux côtières anglaises [20].

\section{2. Échantillonnage}

Les stations (FX7, ..FX11), du transect CherbourgWight (figure 1), ont été échantillonnées périodiquement (septembre et novembre 1994, mars et juillet 1995), à bord des N/O Norôt, Thalia II et Côte d'Aquitaine. Tous les échantillons dits de surface (entre 1 et $2 \mathrm{~m}$ de profondeur) ont été prélevés à l'aide de bouteilles Niskin, ou par l'intermédiaire d'un système de ponpage «tout Téflon ». L'eau de fond ( $2 \mathrm{~m}$ au-dessus de fond) a été prélevée avec

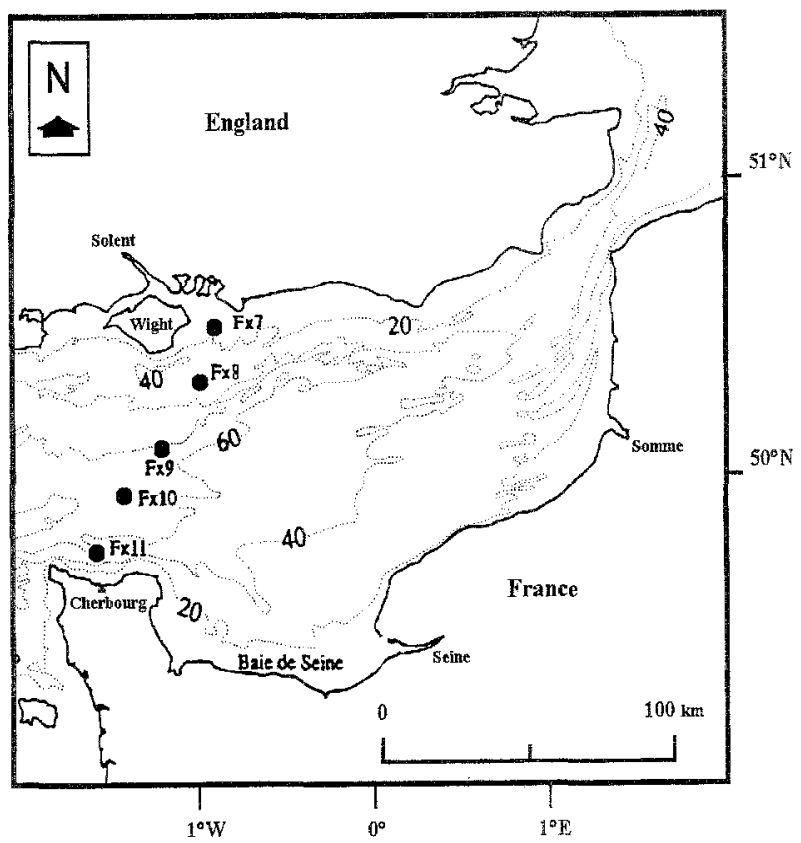

Figure 1. Localisation des stations FluxManche II du transect Cherbourg-Ile de Wight.

Figure 1. Location of FluxManche II sampling stations between the Isle of Wight and Cherbourg. 
des bouteilles Niskin. L'eau de la Seine, prélevée en amont du barrage de Poses [1] et dont les caractéristiques sont les suivantes: salinité : 0,$32 ; \mathrm{COD}: 5,47 \mathrm{mg} \mathrm{L}^{-1}$; $\mathrm{I}_{\mathrm{UFl}}(\lambda \mathrm{ex}=370 \mathrm{~nm}): 54,28 \mathrm{UFl}$, est utilisée comme référence continentale.

Les phases dissoute et particulaire ont été séparées par filtration sous vide, sur filtres «Whatman GF/F » $(0,7 \mu \mathrm{m})$ prépesés et pyrolysés, comme la verrerie utilisée, à $450{ }^{\circ} \mathrm{C}$ pendant 4 heures. L'échantillon destiné à l'analyse du COD est transféré dans un flacon en verre Pyrex d'un volume de $25 \mathrm{~mL}$ (back Téflon) et stérilisé avec $0,12 \mathrm{~mL}$ de $\mathrm{HgCl}_{2}\left(1 \mathrm{~g} \mathrm{~L}^{-1}\right)$. Pour l'analyse spectrofluorimétrique, les échantillons filtrés sont conservés dans des flacons Nalgène (qualité FEP). L'ensemble des échantillons a été conservé à l'obscurité à $4{ }^{\circ} \mathrm{C}$ avant d'être analysé, condition qui permet d'attendre plusieurs dizaines de jours sans qu'une altération notable soit observée.

\subsection{Techniques analytiques}

Les mesures de fluorescence ont été réalisées avec un spectrofluorimètre (Fluorolog Spex 212), muni d'un photomultiplicatcur R928, à doublc monochromateur ct à faible courant d'obscurité. Pour tenir compte de la variation des performances de l'appareil avec le temps, les intensités de fluorescence pour $\lambda \mathrm{ex}=370 \mathrm{~nm}\left(I_{s}\right)$ ont été normalisées en divisant le signal par le rapport des intensités des bandes Raman de l'eau pure (eau Milli-Q) l'une mesurée juste avant chaque échantillon $\left(R_{w, i}\right)$ et l'autre $\left(R_{w, 0}\right)$ prise lors des premières mesures et utilisée comme référence.

$$
\left.I_{n} \text { (intensité normalisée }\right)=I_{s} \frac{R_{w, 0}}{R_{w, t}}
$$

Afin d'être comparables à celles qu'on trouve dans la littérature, ces mesures sont exprimées en unités de fluorescence $(U F l)$ et calculées par référence avec l'intensité $\left(I_{s q}\right)$ de fluorescence du sulfate de quinine $\left(s q, 0,1 \mathrm{mg} \mathrm{L}^{-1}\right.$ dans $\mathrm{HClO}_{4}$ ) mesurée dans les mêmes conditions expérimentales en même temps que $\mathbf{R}_{\mathrm{w}, 0}$. Par définition l'intensité de l'échantillon est exprimée en unités $U F l$ par la relation [10].

$$
I_{U F l}=100 \frac{I_{n}}{I_{s q}}
$$

Dans le cas des spectres enregistrés pour $\lambda e x=313 \mathrm{~nm}$ dont seules les enveloppes spectrales des bandes de fluorescence ont été étudiées, les intensités relatives des maxima ont été normalisées, afin de faciliter leur comparaison avec l'échantillon continental de référence.
Le COD a été mesuré par oxydation catalytique à haute température (OCHT) avec un appareil TOC 5000, Schimadzu, selon le principe élaboré par Sugimura ct Suzuki [21]. Quelques dizaines de microlitres d'échantillon, préalablement décarbonaté par bullage d'oxygène pur pendant 10 min après acidification à $\mathrm{pH} 2$ avec une solution d' $\mathrm{HCl} 2 \mathrm{~N}$, sont oxydés à $680^{\circ} \mathrm{C}$ dans un tube en quartz, en présence de billes d'alumine à $3 \%$ de platine. Le $\mathrm{CO}_{2}$ dégagé par oxydation est quantifié par absorption infrarouge. La concentration est calculée après intégration de la surface des pics et comparaison à des étalons d'hydrogénophtalate de potassium. Les analyses sont effectuées sur au moins trois duplicata et demandent trois à cinq injections, afin d'obtenir un écart type de $2 \%$. Le bullage pouvant entrainer une perte de composés volatils, les valeurs de COD données correspondent à du carbone organique dissous non volatil.

Les données concernant la salinité, la chlorophylle $a$ et les nutriments proviennent des mesures qui nous ont été aimablement communiquées par les participants au programme FluxManche II $[2,4]$.

\section{RÉSULTATS ET DISCUSSION}

\subsection{Variations de la fluorescence à $\lambda \mathrm{ex}=370 \mathrm{~nm}$}

\subsubsection{Intensité de fluorescence}

Pour l'ensemble des échantillons que nous avons étudié (figure 1), les intensités de fluorescence varient entre 2 et 28,6 UFl. A l'exception des stations FX81 en septembre 1994, FX9f et FX11f en juillet 1995, elles décroissent des côtes vers le centre du transect (figure 2). La fluorescence la plus faible est mesurée pour les eaux de la partie centrale (FX9). Ce résultat est en accord avec la nature des masses d'eaux dans cette zone. D'après le modèle numérique de circulation résiduelle [20], la station FX9 est située dans la veine d'eau centrale, venant de l'Atlantique et donc plus pauvre en matière organique fluorescente.

En novembre 1994 et mars 1995, les eaux anglaises fluorescent plus intensément que les eaux françaises. L'abondance de malière organique fluorescente dans les éaux anglaises peut s'expliquer par des apports continentaux importants, en accord avec les salinités plus faibles mesurées pendant ces périodes [4].

En juillet 1995, et contrairement aux autres périodes, les eaux françaises de surface ont un signal de fluorescence comparable, voire légèrement plus intense (station FX11) que celui des eaux anglaises. En l'absence d'un émissaire continental important du côté français, il est possible que 

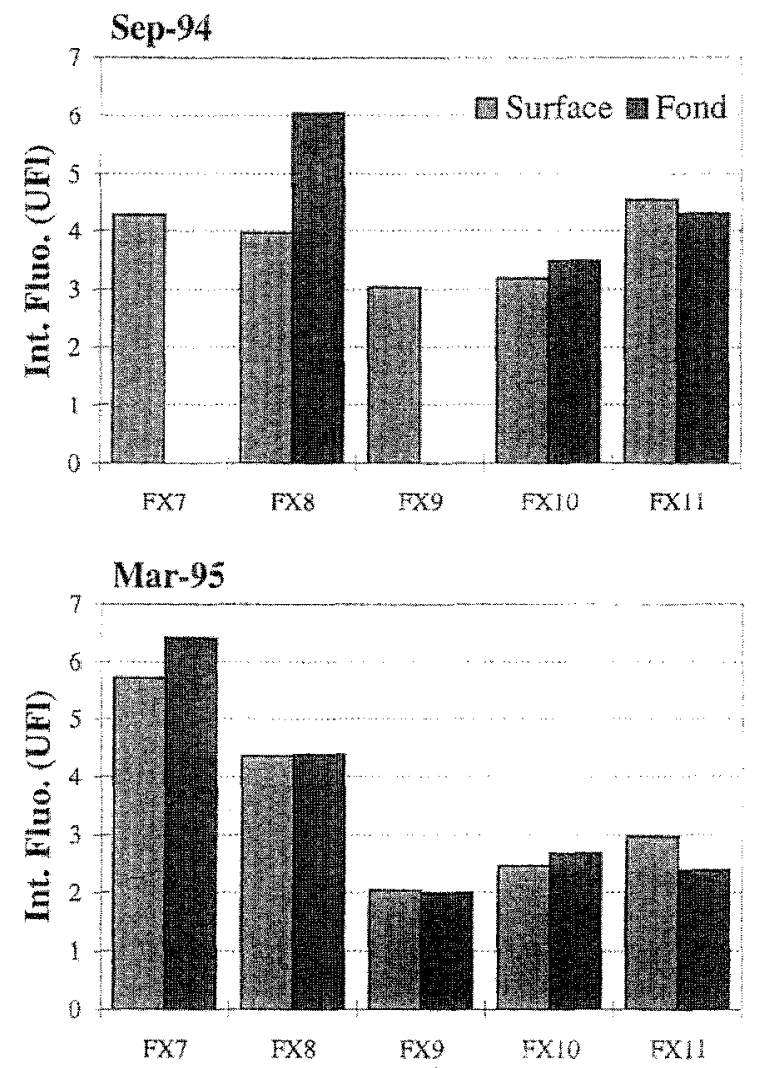
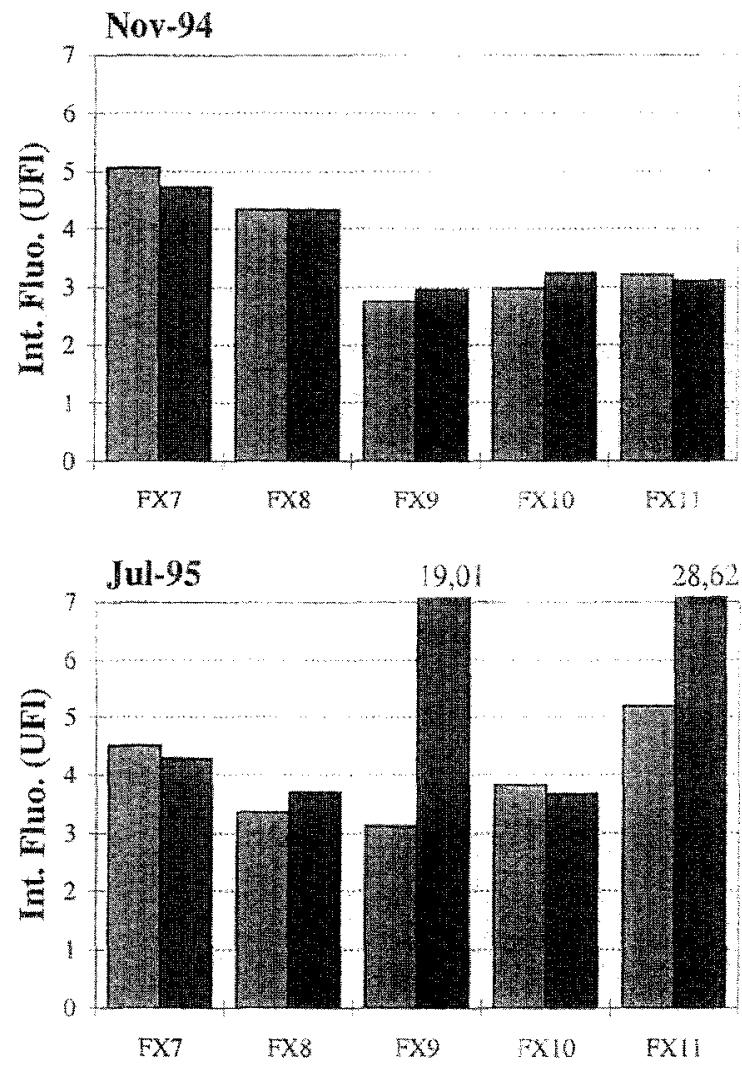

Figure 2. Histogrammes des intensités de fluorescence excitée à $\lambda \mathrm{ex}=370 \mathrm{~nm}$ des eaux du transect Cherbourg-Wight mesurées en septembre et novembre 1994, mars et juillet 1995 .

Figure 2. Spatial distribution of fluorescence intensities of waters of the transect Cherbourg-Isle of Wight measured in September and November 1994, March and July 1995 for an excitation at $\lambda \mathrm{ex}=370 \mathrm{~nm}$.

cette augmentation du matériel lluorescent résulte d'un apport des eaux de la Baie de Seine et/ou du Golfe normand breton, dans lesquels l'influence terrestre est plus marquée. Cela est en accord avec une diminution de la salinité à cette station et avec une concentration en chlorophylle a rclativement plus élevée $\left(1,5 \mu \mathrm{L}^{-1}\right)$ qu'aux autres stations. Les intensités anormalement élevées au fond des stations FX9 et FX11 ( 7 à 8 fois) restent inexpliquées. Il pourrait s'agir d'une pollution ponctuelle.

\subsubsection{Relation intensité de fluorescence-salinité}

Pour les quatre périodes d'étude, une corrélation linéaire négative est observée entre l'intensité de fluorescence et la salinité, avec des coefficients de corrélation respectifs de $r^{2}=0,85$ (excepté FX8f) $r^{2}=0,94$ (excepté FX9s); $r^{2}=0,94$ et $r^{2}=0,85$ (exceptés FX9f et FX11f) (figure 3). Les points présentant un écart à la relation sont probablement liés à des anomalies ponctuelles. Celles-ci se mani- festent soit par un écart d'intensité non négligeable entre la surface et le fond, soit par une anomalie de salinité, comme dans le cas de FX9s en novembre. La corrélation négative observée semble résulter d'une dilution du matériel organique fluorescent, des côtes vers le centre du transect. Cela suggère que ce matériel fluorescent aurait pour origine un composant terrestre riche en substances humiques, en accord avec Moran et Hodson [18] et Klinkhammer et al. [16] qui indiquent que dans les eaux côtières, le matériel terrestre se trouve préférentiellement sous forme de substances humiques dans le COD marin.

Le long du transect Cherbourg-Wight, en accord avec la distribution de la salinité et la répartition du matériel fluorescent, on distingue trois types de masses d'eaux : 1) les eaux côtières britanniques relativement moins salées et en moyenne (4-5 UFl) les plus riches en substances humiques ; 2) les eaux côtières trançaises caractérisées par une fluorescence intermédiaire (3-4 UFl) ; 3) entre 


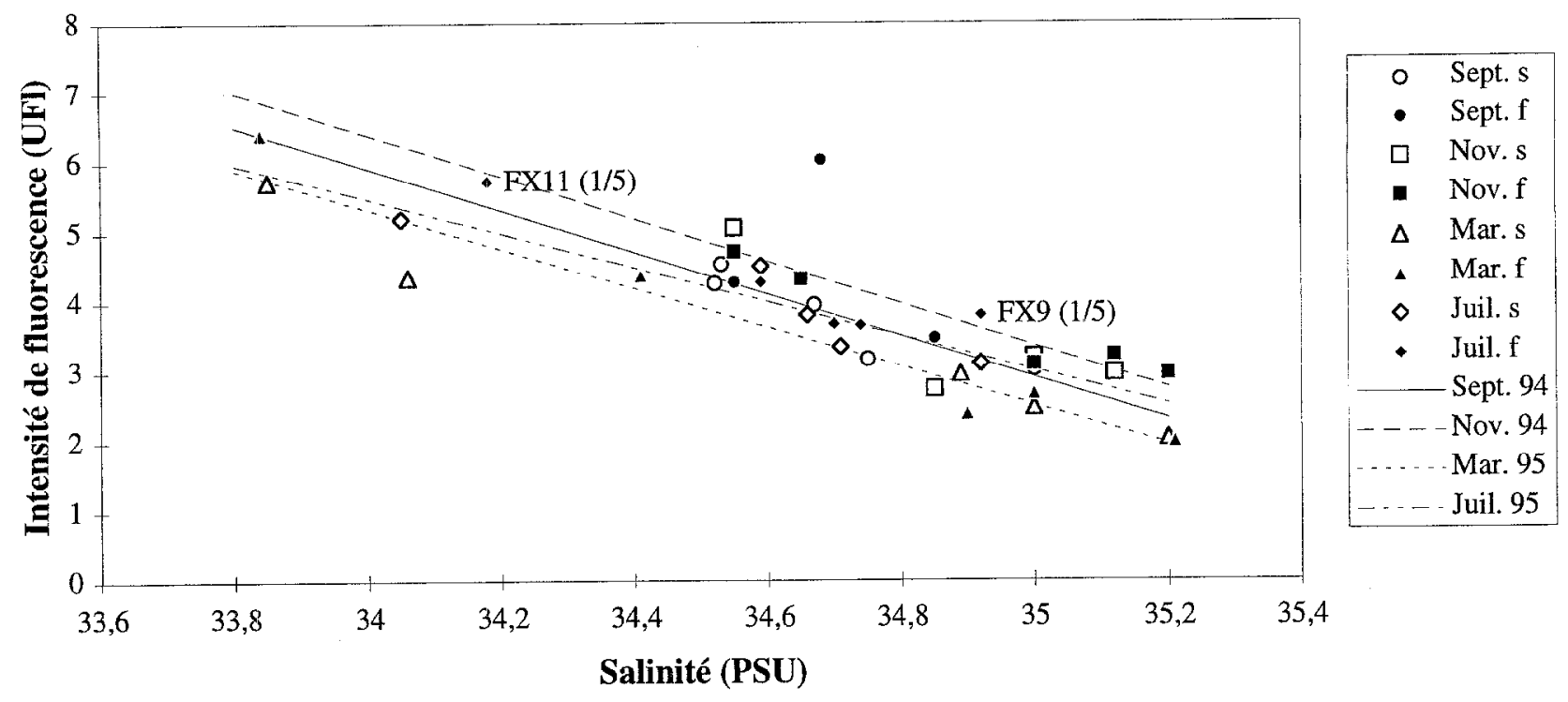

Figure 3. Relations entre les intensilés de fluorescence des eaux excitée à $\lambda$ ex $=370$ nn el la salinité pour les radiales de septenıbre et novennbre 1994, mars et juillet 1995. Les symboles creux et pleins correspondent respectivement aux échantillons de surface (s) et de fond (f) des stations de prélèvement. Les intensités des échantillons FX9f et FX11f en juillet 1995 ont été divisés par 5.

Figure 3. Relationships between fluorescence intensity of waters excited at $\lambda \mathrm{ex}=370 \mathrm{~nm}$ and the salinity for the radials of September and November 1994, March and July 1995. Open and filled symbols correspond respectively to surface (s) and bottom (f) sampling stations. Intensities of samples FX9f and FXI If in July 1995 has been divided by 5 .

ces deux masses d'eaux côtières, circule une masse d'eau plus salée et moins fluorescente $(\approx 2,5 \mathrm{UFl})$ et présentant le caractère marin le plus prononcé, en raison de l'influence plus marquée des eaux d'origine atlantique, ce qui est en accord avec la structure des masses d'eaux le long du transect.

\subsubsection{Relation intensité de fluorescence - $C O D$}

Dans le cas des eaux du transect, le COD n'est corrélé ni avec la salinité, ni avec la chlorophylle a (tableau I). En effet, le matériel organique dissous dans son ensemble se répartit sans gradient particulier, reflétant la diversité de ses origines [15]. Cela est à rapprocher des résultats récents de Bodineau et al. [3], qui montrent que le COD des eaux du transect se caractérise par des variations saisonnières complexes, sans tendance particulière. La fluorescence n'est pas corrélée au COD (tableau I). Dans les eaux océaniques, une corrélation approximative entre la concentration du COD et la fluorescence a été notée par Chen et Bada [6], mais elle serait négative. L'absence de corrélation entre l'intensité de fluorescence pour $\lambda$ ex $=370 \mathrm{~nm}$ et le COD indique qu'une partie du COD marin correspond à des substances qui sont à la fois dif- férentes des $\mathrm{SH}$ et non corrélées temporellement et spatialement avec ces dernières.

\subsubsection{Relation intensité de fluorescence - sels nutritifs}

Les valeurs disponibles des concentrations en nutriments correspondent aux transects de mars et juillet 1995 [2].

Il est intéressant de constater que la distribution des sels nutritifs se corrèle assez bien avec l'intensité de fluorescence. Les coefficients de corrélation $\left(r^{2}\right)$ sont pour $\mathrm{Si}$, $\mathrm{NO}_{3}^{-}, \mathrm{PO}_{4}^{3-}$, respectivement de $0,95,0,92,0,84$ en mars et $0,46,0,68,0,79$ en juillet (figure 4). Cette corrélation observée dans les eaux de surface et de fond du transect, traduit un comportement similaire de ces paramètres. Des apports continentaux en nutriments et en matériel fluorescent sont observés dans les eaux côtières, notamment du côté anglais. Les eaux centrales à caractère plus marin, sont faiblement enrichies en ces deux substances. Il est probable qu'une régénération des sels nutritifs avec production de matériel fluorescent accompagne le recyclage de la matière organique autochtone. Le fait que la relation entre les deux paramètres soit moins bonne en juillet qu'en mars peut être imputé aux processus physiques et biologiques qui prévalent en cette période [14] et qui 
Tableau I. Coefficient de corrélation $\left(r^{2}\right)$ el niveau de confiance $(p)$ pour les relations fluorescence-COD, COD-sadinité et COD-chlorophylle $a$.

Table I. Coefficient of determination $\left(r^{2}\right)$ and confidence interval $(\rho)$ for relationships between fluorescence-DOC, DOC-salinity and DOCchlorophyll.

\begin{tabular}{lccccccccc}
\hline & \multicolumn{3}{c}{ Fluo. vs COD } & \multicolumn{3}{c}{ COD vs Salinité } & \multicolumn{2}{c}{ COD vs Chl. $\boldsymbol{a}$} \\
\hline & $\boldsymbol{r}^{\mathbf{2}}$ & $\rho$ & $\boldsymbol{n}$ & $\boldsymbol{r}^{\mathbf{2}}$ & $\rho$ & $\boldsymbol{n}$ & $\boldsymbol{r}^{\mathbf{2}}$ & $\rho$ & $\boldsymbol{n}$ \\
\hline Sept. 94 & 0,014 & 0,77 & 8 & 0,034 & 0,60 & 10 & 0,065 & 0,47 & 10 \\
Nov. 94 & 0,226 & 0,16 & 10 & 0,084 & 0,41 & 10 & 0,162 & 0,24 & 10 \\
Mar. 95 & 0,026 & 0,65 & 10 & 0,005 & 0,84 & 10 & 0,131 & 0,30 & 10 \\
Juil. 95 & 0,049 & 0,538 & 10 & 0,105 & 0,36 & 10 & 0,000 & 0,98 & 10 \\
\hline
\end{tabular}

aboutissent à une importante variabilité spatiale des teneurs en nutriments.

\subsection{Variations de la fluorescence à $\lambda \mathrm{ex}=313 \mathrm{~nm}$ et $\lambda \mathrm{ex}=270 \mathrm{~nm}$}

\subsubsection{Caractéristiques spectrales}

Par rapport au spectre de la référence continentale (Ref) et quelle que soit la période, les spectres de fluorescence des eaux du transect présentent deux élargissements, l'un vers le rouge disparaît dans certains cas (FX11s en mars 1995 et $\mathrm{FX}_{\mathrm{s}}$ en juillet 1995) et l'autre vers le bleu est présent dans tous les échantillons (figures 5 et 6 ). Du côté des courtes longueurs d'onde, l'élargissement s'accompagne d'un épaulement, voire d'un deuxième maximum (FX8s en juillet 1995) (figure 6). Des spectres similaires sont observés par Belin et al. (1997) sur des échantillons côtiers de la Manche orientale et traduisent la contribution d'une composante (b) vers le bleu, prédominante dans les eaux marines et celle d'une composante (a) située vers le rouge et qui marquerait la présence de matériel humique continental.

Généralement, il a été observé que les eaux purement marines ont des spectres nettement décalés vers le bleu par rapport aux eaux continentales $[6,8,10,12]$. Dans le (a)
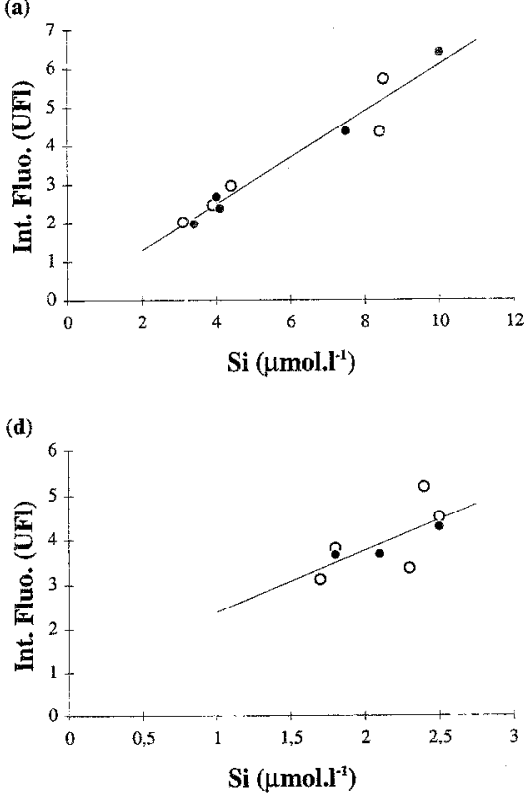

(b)

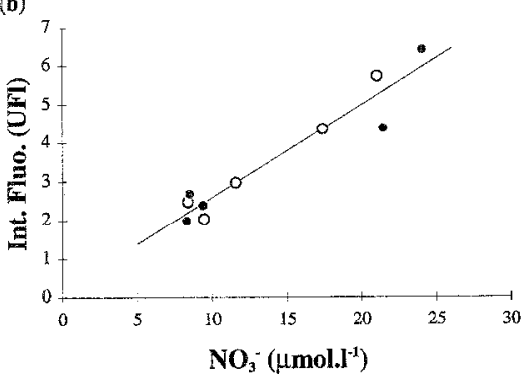

(e)

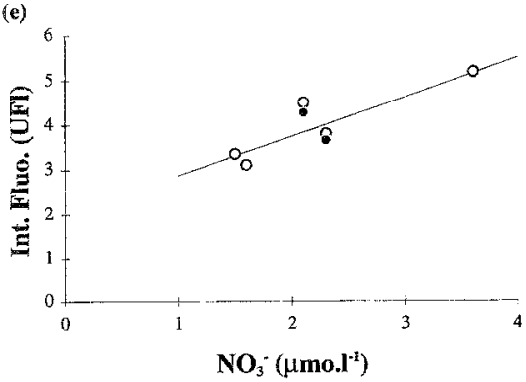

(c)

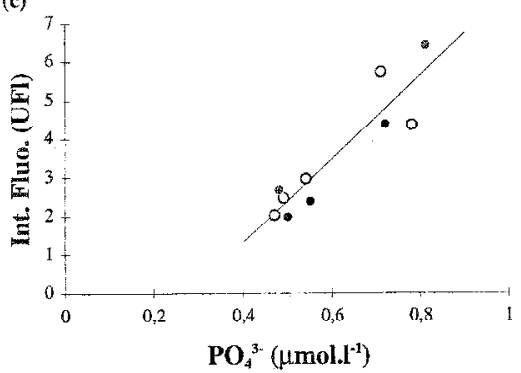

(f)

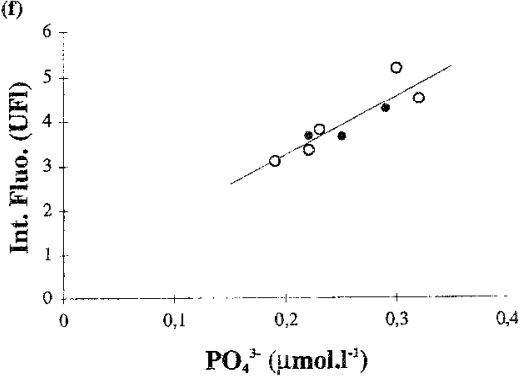

Figure 4. Relations entre la fluorescence excitée à $\lambda \mathrm{ex}=370 \mathrm{~nm}$ et les nutriments dans les eaux du transect pour les radiales de mars 1995 (a b, c) et juillet 1995 (d, e, f). (o) : échantillons de surface ; $(\bullet)$ : échantillons de fond.

Figure 4. Relationships between the fluorescence excited at $\lambda \mathrm{ex}=370 \mathrm{~nm}$ and the nutrients in waters of the transect for radials of March 1995 $(\mathbf{a}, \mathbf{b}, \mathbf{c})$ and July 1995 (d, e, f). (o): surface samples; (•): bottom samples. 
cas de la Manche, ce déplacement du maximum d'émission vers les courtes longueurs d'ondes est peu apparent, en raison de l'importance de la composante $a$ due principalement aux substances humiques continentales.

Les résultats obtenus sur les échantillons du transect confirment ceux de Belin et al. [1], en particulier sur la réponse spectrale des eaux de la Manche et la présence de deux familles distinctes de chromophores dont l'une (composante $b$ ) sernble caractériser le milieu marin.

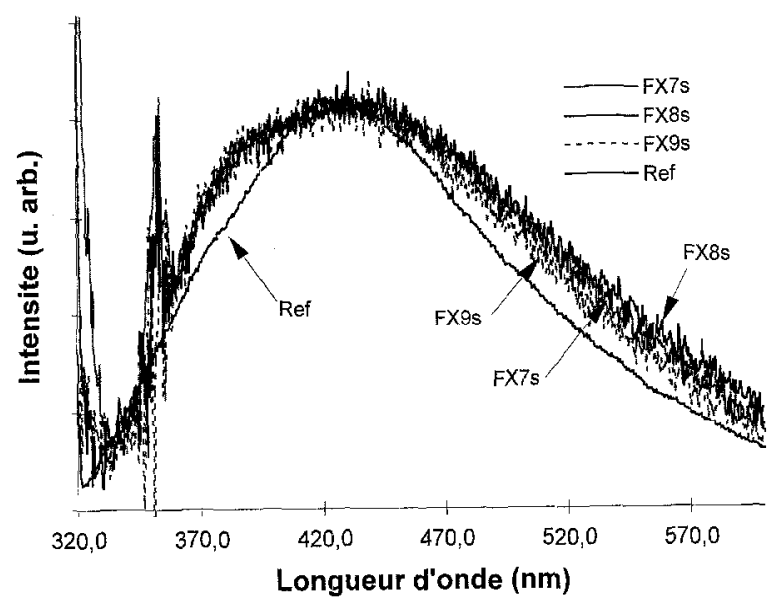

(a)

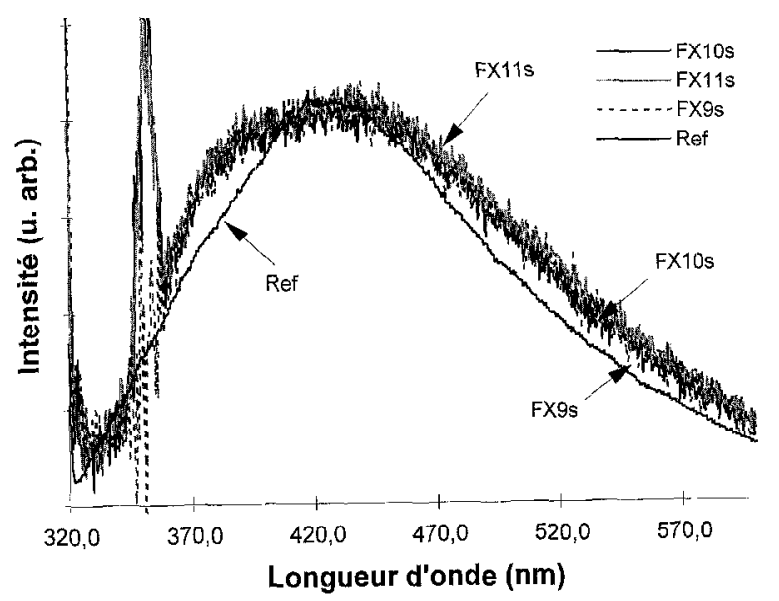

(b)

Figure 5. Spectres de fluorescence excitée à $\lambda \mathrm{ex}=313 \mathrm{~nm}$ des échantillons de surface des eaux du transect prélevés lors de la radiale de septembre 1994, comparés au spectre de la référence continentale (Ref). Pour faciliter la comparaison les spectres sont normalisés au même maximum

Figure 5. Fluorescence spectra excited at $\lambda \mathrm{ex}=313 \mathrm{~nm}$, for surface waters of the transect compared to the spectrum of the continental reference (Ref) in the case of the radial of September 1994. To facilitate the comparison the spectrum maxima are normalized.

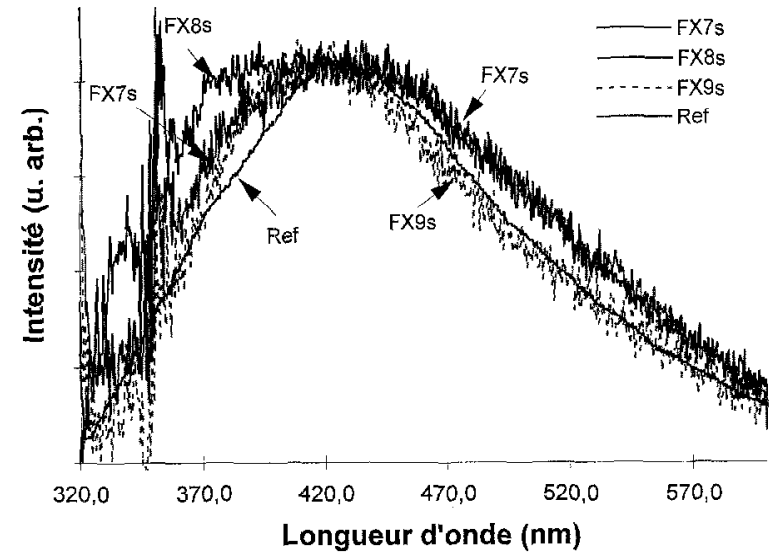

(a)

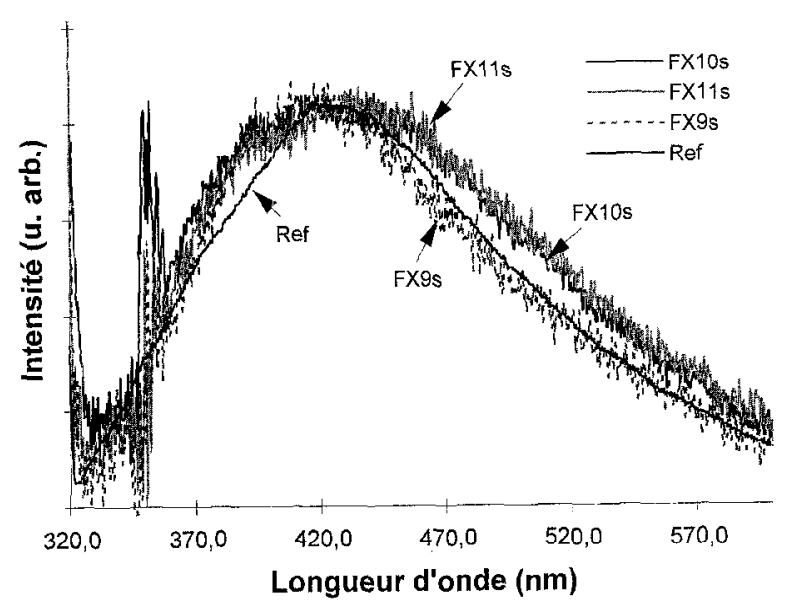

(b)

Figure 6. Spectres de fluorescence excitée à $\lambda \mathrm{ex}=313 \mathrm{~nm}$ des eaux de surface du transect Cherbourg-Wight comparés au spectre de la référence continentale (Ref) dans le cas de la radiale de juillet 1995.

Figure 6. Fluorescence spectra excited at $\lambda \mathrm{ex}=313 \mathrm{~nm}$, for surface waters of the transect Cherbourg-Isle of Wight compared to the spectrum of the continental reference (Ref) in the case of the radial of July 1995.

\subsubsection{Variations spatio-temporelles}

Seules les variations spatio-temporelles des spectres de fluorescence des échantillons prélevés en surface sont discutées ici, en raison des similitudes spectrales entre la surface et le fond.

En septembre 1994, tous les spectres présentent un élargissement semblable, à la fois du côté des grandes et des courtes longueurs d'onde, ce qui indique le caractère relativement homogène des eaux le long du transect 


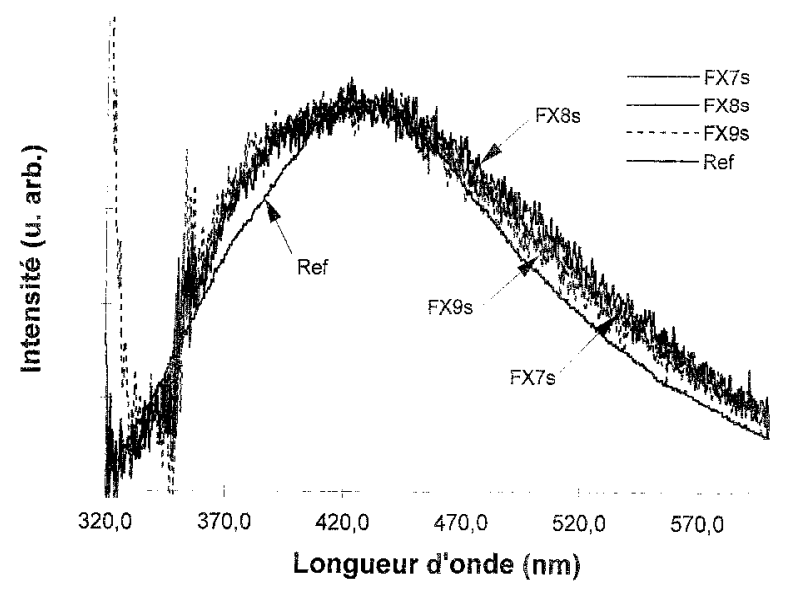

(a)

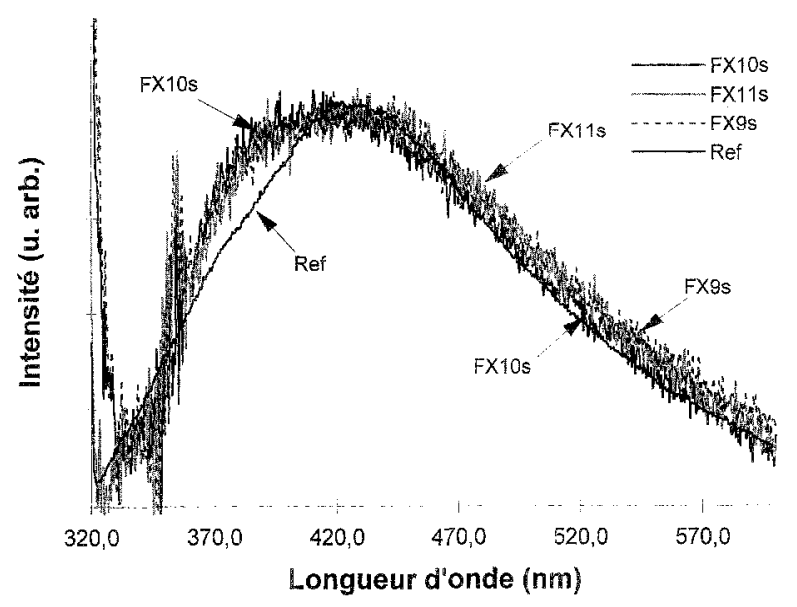

(b)

Figure 7. Comparaison des profils des spectres de fluorescence pour $\lambda_{\mathrm{ex}}=313 \mathrm{~nm}$, de l'ćchantillon continental de réfćrence (Ref) et des spectres des eaux de surface prélevées lors de la radiale de novembre 1994.

Figure 7. Comparison of the fluorescence spectra profiles for $\lambda \mathrm{ex}=313 \mathrm{~nm}$, of the continental reference sample (Ref) and the spectra of surface waters collected during the radial of November 1994.

(figure 5). En novembre 1994 et mars 1995, les spectres des stations FX7, FX8 sont caractérisés par un élargissement légèrement plus important du côté des grandes longueurs d'onde, par comparaison avec les autres stations (figures 7 et 8). Cet élargissement peut s'expliquer par un apport de matériel humique terrestre, qui traduit l'influence des eaux du système Avon-Solent.

En juillet 1995, à l'exception de celui de la station FX9s, les spectres montrent un élargissement similaire du côté des grandes longueurs d'onde qui indique la présence de matériel organique d'origine continentale dans les zones

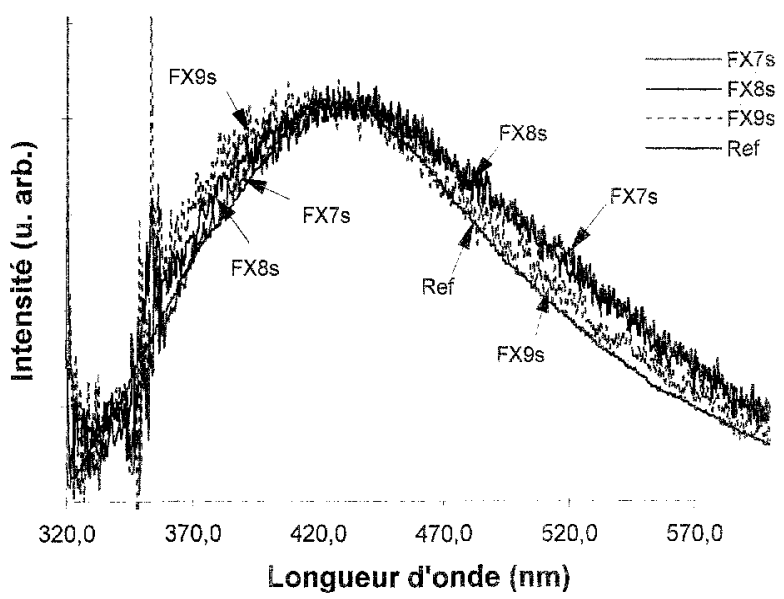

(a)

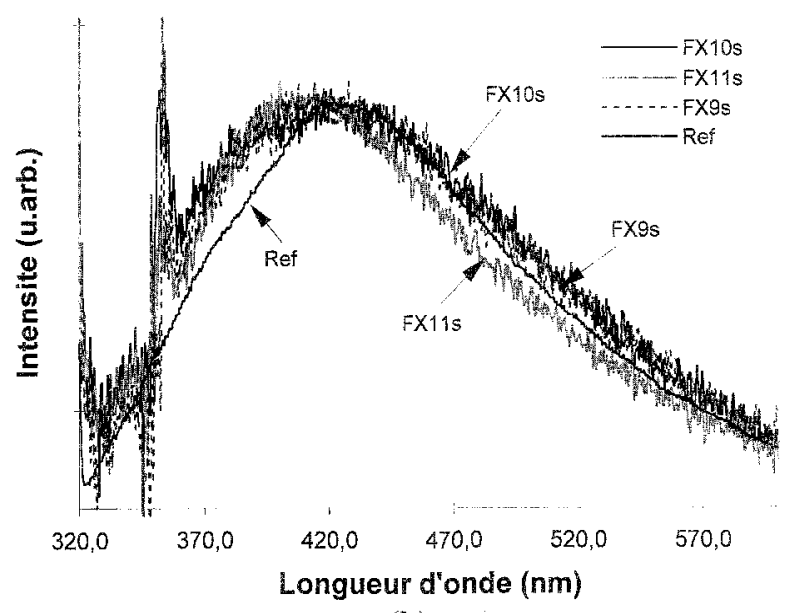

(b)

Figure 8. Comparaison des spectres de fluorescence pour $\mathrm{I}_{\mathrm{ex}}=313 \mathrm{~nm}$, de l'échantillon continental de référence (Ref) et des spectres des eaux de surface prélevées lors de la radiale de mars 1995.

Figure 8. Comparison of the fluorescence spectra profiles for $\lambda e x=31.3 \mathrm{~nm}$, of the continental reference sample (Ref) and the spectra of surface waters collected during the radial of March 1995.

côtières (figure 6). En revanche, la composante b, présente dans tous les échantillons, est particulièrement intense dans le spectre de la station FX8s, ce qui semble indiquer la présence de matériel organique marin autochtone qui pourrait être lié au développement d'une activité biologique locale.

\subsubsection{Spectres de fluorescence pour $\lambda \mathrm{ex}=270 \mathrm{~nm}$}

L'excitation de la fluorescence des eaux naturelles à $\lambda \mathrm{ex}=270 \mathrm{~nm}$ fait apparaitre, outre la bande caractéristi- 


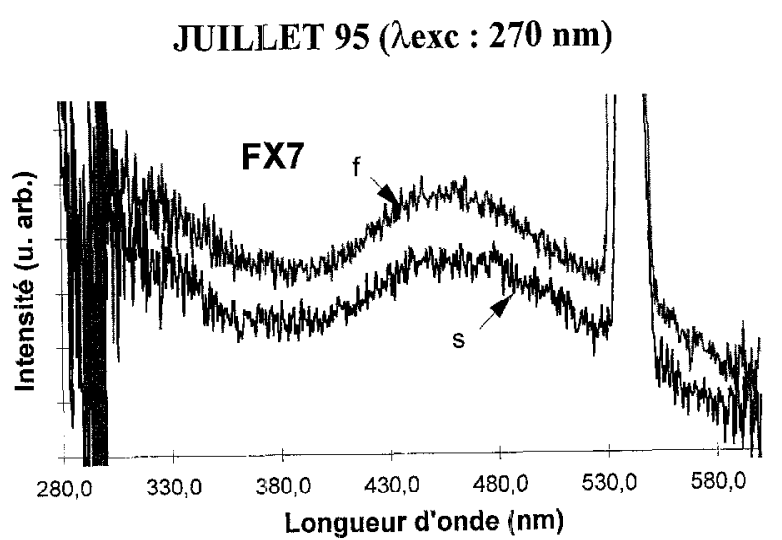

(a)

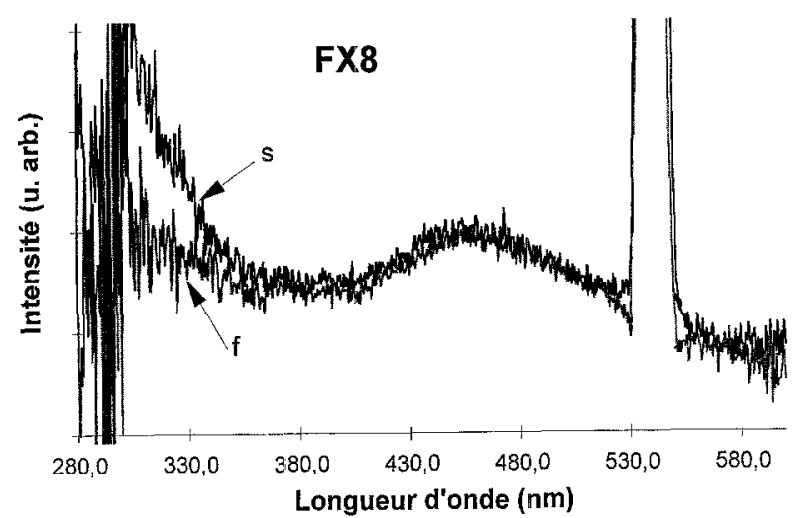

(b)

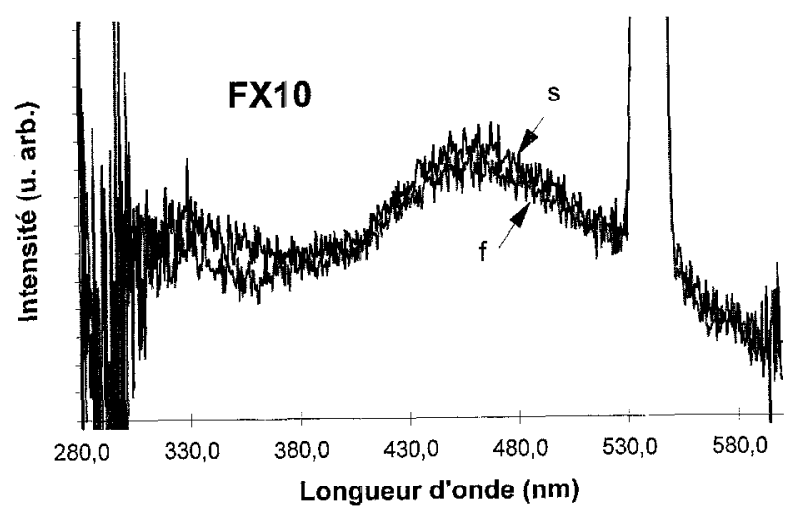

(c)

Figure 9. Spectres de fluorescence pour $\lambda \mathrm{ex}=270 \mathrm{~nm}$ des eaux des stations FX7, FX8 et FX10 (s : surface, $\mathrm{f}$ : fond) prélevées lors de la radiale de juillet 1995 . La bande observée autour de $300 \mathrm{~nm}$ indique la présence de fluorophores de type protéinique (voir texte). La bande très large à $\lambda \max \approx 460 \mathrm{~nm}$ est due aux substances humiques.

Figure 9. Fluorescence spectra for $\lambda \mathrm{ex}=270 \mathrm{~nm}$ of station waters FX7, FX8 and FX10 (s: surface, f: bottom) collected during the radial of July 95 . The band observed around $300 \mathrm{~nm}$ indicate the presence of fluorophores protein-like (see text). The very large band observed at $\lambda \max \approx 460 \mathrm{~nm}$ correspond to humic substance. que des substances humiques $(\lambda \max =460 \mathrm{~nm})$, une bande dont le maximum se situe vers 310-330 nm. Dans la littérature, celle-ci est attribuée à la fluorescence d'acides aminés (tyrosine ou tryptophane) libres ou liés à des résidus protéiniques provenant d'exsudats de matériel biologique ou de leur dégradation récente $[9,13,17]$.

Quelle que soit la période, cette bande n'est visible qu'en septembre 1994 dans l'échantillon. FX8f et, à l'exception de FX9f et FX11f, dans tous les échantillons de surface et de fond en juillet 1995, avec des intensités relativement faibles (figure 9). Belin et al. [1] observent cette même bande en baie de Seine et le long du littoral français de la Manche orientale, dans la structure hydrologique particulièrement productive du «fleuve côtier» [5], avec des intensités plus élevées que celles rapportées ici. Les faibles intensités de la bande protéinique dans les eaux du transect pourraient être liées à une productivité primaire plus faible qu'en Manche orientale. Compte tenu de la bioréactivité et de la photoréactivité des substances protéiniques libérées sous forme dissoute [17], les faibles intensités observées pourraient s'expliquer par un décalage temporel trop important entre les poussées phytoplanctoniques printanières, pendant lesquelles les substances sont libérées, et nos prélèvements de mars et juillet 1995 [2, 41. Ces résultats sont en accord avec ceux de Chen et Bada [6], qui n'observent pas de corrélation entre la fluorescence des eaux marines de surface et la productivité.

\section{CONCLUSION}

Les paramètres de fluorescence exploités dans cette étude, ont permis de caractériser la matière organique dissoute fluorescente dans les eaux du transect Cherbourg-Wight.

La relation liant la fluorescence $(\lambda \mathrm{ex}=370 \mathrm{~nm})$ à la salinité, traduit la dilution du matériel humique fluorescent des côtes vers le centre du transect. Ce comportement conservatif du signal de fluorescence permet de l'utiliser comme traceur sensible, capable de détecter les substances humiques dans les eaux marines et de visualiser leur répartition entre les différentes masses d'eaux. Les zones côtières, en particulier les eaux anglaises, sont caractérisées par les teneurs les plus élevées en substances humiques. C'est la conséquence des apports continentaux du Solent. Le faible signal de fluorescence des eaux centrales témoigne de leur caractère marin peu ou pas influencé par des apports terrestres. 
L'excitation de la fluorescence à 313 num mel en évidence dans la signature spectrale des eaux étudiées, à la fois la contribution d'une composante d'origine marine et celle d'une composante d'origine continentale. La bande de fluorescence relative aux substances dites de type protéinique est de faible intensité. Ce résultat pourrait refléter soit une production primaire plus faible qu'en Manche orientale, soit le fait que les échantillons ont été prélevés en dehors de la floraison phytoplanctonique.

\section{Remerciements}

Ce travail a été effectué dans le cadre du programme européen Mast II (Fluxmanche II, Contrat MAS2 - CT940089). Les auteurs remercient L. Cabioch et M. Wartel, coordinateurs successifs et P.J. Statham, responsable du thème Flux et processus géochimiques. Ils remercient les équipages des N/O: Norô̂t, Thalia II et Côte d'Aquitaine, ainsi que Mme A. Hurel, technicienne au laboratoire de Géologie marine de l'Université de Caen.

\section{RÉFÉRENCES}

[1] Belin C. Lamotte M., Ewald M., Texier H., Fluorometric characterization of the Seine estuary, Seine bay and French coastal Channel waters, Estuar. Coast. Shelf Sci. (1997) (sous presse).

[2] Bentley D., Hart V., Guary J.C., Statham P.J., Task G., Nutrient fluxes in the central Channel, Final report FluxManche II (1996) 180-191.

[3] Bodineau L., Thoumelin G., Wartel M., Task G., Seasonal fluxes and changes in composition of organic matter in the eastern Channel, Final report FluxManche II (1996) 192-204.

[4] Boxall S., Bishop C., Nash L., Santer R., Chami M., Dilligeard E., Wernand M., Matthews A., Task W., Physical processes and field measurements, Final report FluxManche II (1996) 53-72.

[5] Brylinski J.-M, Lagadeuc Y., Gentilhomme V., Dupont J.-P., Lafite R., Dupeuble P.-A., Huault M.-F., Auger Y., Puskaric E., Wartel M., Cabioch L., Le "Fleuve côtier" : un phénomène hydrologique important en Manche orientale, Exemple du Pasde-Calais, Oceanol. Acta, vol. sp. 11 (1991) 197-203.

[6] Chen R.F., Bada J.L., The fluorescence of dissolved organic matter in seawater, Mar. Chem. 37 (1992) 191-221.

[7] Coble P.G., Characterization of marine and terrestrial DOM in seawater using excitation-emission matrix spectroscopy, Mar. Chem. 51 (1996) 325-346.

[8] Coble P.G., Brophy M.M., Investigation of the geochemistry of dissolved organic matter in coastal waters using optical properties. SPIE, Ocean Opics XII (2258) (1994) 377-389.

[9] Coble P.G., Green S.A., Blough N.V., Gagosian R.B., Characterization of dissolved organic matter in the Black Sea by fluorescence spectroscopy, Nature 348 (1990) 432-435.

[10] de Souza-Sierra M.M., Donard O.F.X., Lamotte M., Belin C., Ewald M., Fluorescence spectroscopy of coastal and marine waters, Mar. Chem. 47 (1994) 127-144.

[11] Determann S., Reuter R., Wagner P., Willkomm R., Fluorescent matter in the eastern Atlantic Ocean, Part 1: Methods of measurement and near-surface distribution, Deep-Sea Res. 41 (1994) 659-675.
[12] Donard O.F.X., Lamotte M., Belin C., Ewald M., High sensitivity fluorescence spectroscopy of mediterranean waters using a conventional or pulsed laser excitation source, Mar. Chem. 27 (1989) 117-136.

[13] Ewald M., Stabel H.H., Belin C., Composés organiques d'origine biogénique dans l'eau de la zône euphotique en Antarctique étudiés directement par spectrofluorimétrie, C. R. Acad. Sci. Paris 302, série II, 14 (1986) 883-886.

[14] Hart V., Statham P.J., Task G., Distribution, fluxes and processes influencing nutrients in the Solent and adjacent waters during FluxManche II, and dissolved metals in this region. Final report FluxManche II (1996) 238-251.

[15] Ismaîli M.M., Traceurs dissous et particulaires de la matière organique dans l'Estuaire de la Seine, la Baie de Seine et la Manche centrale, Thèse de doctorat, université de Caen, 1998, $180 \mathrm{p}$.

[16] Klinkhammer G.P., Chin C.S., Wilson C., Rudnicki M.D., German C.R., Distributions of dissolved manganese and fluorescent dissolved organic matter in the Columbia River estuary and plume as determined by in situ measurement, Mar. Chem. 56 (1997) 1-14.

[17] Mopper K., Schultz C.A., Fluorescence as a possible tool for studying the nature and water column distribution of DOC Components, Mar. Chem. 41 (1993) 229-238.

[18] Moran M.A., Hodson R.E,, Dissolved humic substances of vascular plant origin in a coastal marine environment, I,imnol. Oceanogr. 39 (4) (1994) 762-771.

[19] Salomon J.C., Quelques aspects hydrodynamiques de la Manche. Equinoxe : Les ressources vivantes de la mer et l'environnement littoral, Equinoxe 47-40 (1994) 68-73.

[20] Salomon J.C., Breton M., Courants résiduels de marée dans la Manche, Oceanol. Acta, Proceedings of the International Colloquium on the environment of epicontinental seas, Lille, 20-22 March, vol. sp. 11 (1991) 47-53.

[21] Sugimura Y., Suzuki Y., A high temperature catalytic oxidation method of non-volatile dissolved organic carbon in seawater by direct injection of a liquid sample, Mar. Chem. 24 (1988) 105-131. 